\title{
Intact implicit statistical learning in borderline personality disorder
}

\author{
Zsolt Unoka ${ }^{\mathrm{a}, 1}$, Gabriella Vizin ${ }^{\mathrm{a}}$, Anna Bjelik ${ }^{\mathrm{a}}$, Dóra Radics ${ }^{\mathrm{a}}$, Dezso Nemeth ${ }^{\mathrm{b}, \mathrm{c}, *, 1}$, \\ Karolina Janacsek ${ }^{\mathrm{b}, \mathrm{c}, 1}$ \\ a Department of Psychotherapy and Psychiatry, Semmelweis University, Budapest, Hungary \\ b Institute of Psychology, Eötvös Loránd University, Izabella utca 46., H-1064 Budapest, Hungary \\ ${ }^{\mathrm{c}}$ MTA-ELTE NAP B Brain, Memory and Language Research Group, Institute of Cognitive Neuroscience and Psychology, Research Centre for Natural Sciences, Hungarian \\ Academy of Sciences, Magyar tudósok körútja 2., H-1117 Budapest, Hungary
}

\section{A R T I C L E I N F O}

\section{Keywords:}

Borderline Personality Disorder

Implicit learning

Statistical learning

Skill learning

\begin{abstract}
A B S T R A C T
Wide-spread neuropsychological deficits have been identified in borderline personality disorder (BPD). Previous research found impairments in decision making, declarative memory, working memory and executive functions; however, no studies have focused on implicit learning in BPD yet. The aim of our study was to investigate implicit statistical learning by comparing learning performance of 19 BPD patients and 19 healthy, age-, education- and gender-matched controls on a probabilistic sequence learning task. Moreover, we also tested whether participants retain the acquired knowledge after a delay period. To this end, participants were retested on a shorter version of the same task $24 \mathrm{~h}$ after the learning phase. We found intact implicit statistical learning as well as retention of the acquired knowledge in this personality disorder. BPD patients seem to be able to extract and represent regularities implicitly, which is in line with the notion that implicit learning is less susceptible to illness compared to the more explicit processes.
\end{abstract}

\section{Introduction}

Borderline personality disorder (BPD) is present in approximately $0.7-2.7 \%$ of the general population, about $6 \%$ in primary care settings, about $10 \%$ among individuals seen in outpatient mental health clinics, and about 20\% among psychiatric inpatients (APA, 2013; Coid et al., 2006; Grant et al., 2008; Trull et al., 2010). BPD compared to other personality disorders is characterized by the enduring patterns of more dysphoric and less positive cognitive and affective states (Reed et al., 2012a, 2012b). BPD patients are also characterized by rigid, early maladaptive schemas (Unoka et al., 2011) that negatively distorts social cues (Richman and Unoka, 2015). Their distorted interpersonal perception leads to negative affects (Sadikaj et al., 2010) and maladaptive interpersonal functioning (Stepp et al., 2009) that are inflexible and pervasive across a broad range of personal and social situations (Gunderson et al., 2011). Exploration of cognitive functions, such as executive/control functions, learning and memory can help better understand these alterations in social perception and behavior.

Neuropsychological deficits have been identified as a core feature of BPD and are a central manifestation of the pathophysiology of the disorder (Unoka and Richman, 2016). In a recent meta-analysis (Unoka and Richman, 2016) it was found a large overall effect size for global cognition deficit in BPD, with a large effect size for decision making, memory and executive functioning, and a small effect size for visuospatial abilities, attention, and verbal intelligence and processing speed (Beblo et al., 2006; Black et al., 2009; Haaland et al., 2009; Haaland and Landrø, 2007; LeGris et al., 2012; Minzenberg et al., 2008; Rentrop et al., 2007; Richman and Unoka, 2015; Ruocco, 2005; Seres et al., 2009). Although previous studies have focused on several aspects of declarative/explicit learning and memory (e.g., recognition and recall, the effect of emotions on learning) (Ebner-Priemer et al., 2009), to best of our knowledge, no study has investigated non-declarative, implicit learning yet.

Implicit learning occurs when predictive relationships in form of statistical regularities or sequence of events are extracted from the environment without putting conscious effort into the process or realizing the learning process at all (Reber, 1993). Research has shown that implicit learning plays a critical role in guiding our behavior in many day-to-day activities; it is involved in obtaining not only motor skills (e.g., mastering sports), but in learning and processing languages (Kaufman et al., 2010; Nemeth et al., 2011; Rebuschat, 2013), in learning to play a musical instrument or in perceiving music (Rohrmeier and Rebuschat, 2012; Romano Bergstrom et al., 2012), as well as in social learning and social skills (Lieberman, 2000; Norman

\footnotetext{
* Corresponding author at: Institute of Psychology, Eötvös Loránd University, Izabella utca 46., H-1064 Budapest, Hungary.

E-mail address: nemeth@nemethlab.com (D. Nemeth).

${ }^{1}$ These authors contributed equally to the paper.
} 
and Price, 2012). The aim of our study here is to test, for the first time, whether implicit statistical learning is intact or impaired in BPD.

In our study we used a classical implicit learning task, namely the Alternating Serial Reaction Time (ASRT) task, to measure implicit learning of statistical regularities (Howard and Howard, 1997; Janacsek et al., 2012). Moreover, we aimed to test not only the learning of these statistical regularities but also whether BPD patients can successfully retain the acquired knowledge after a delay. It has widely been shown that implicit learning relies mainly on the fronto-striatal networks (Daw et al., 2005; Doyon et al., 2009; Hikosaka et al., 1999; Janacsek et al., 2012; Poldrack et al., 2005; Reber, 2013). Previous studies have found dissociation between implicit learning/retention and other forms of learning/memory in other clinical populations, showing intact implicit learning/retention and impairments in short-term, working and declarative memory in the same group of patients (e.g., patients with sleep disorders, (Csabi et al., 2015, 2014; Virag et al., 2015)). Those findings suggest that implicit learning/memory might be less susceptible to illness compared to other forms of learning/memory (Reber, 1993; Reber and Allen, 2000; Reber, 2013).

In summary, alterations in executive functioning, declarative/explicit memory and decision making has widely been reported in BPD (Beblo et al., 2006; Black et al., 2009; Haaland et al., 2009; Haaland and Landrø, 2007; LeGris et al., 2012; Minzenberg et al., 2008; Rentrop et al., 2007; Richman and Unoka, 2015; Ruocco, 2005; Seres et al., 2009). In contrast, non-declarative, implicit learning has been neglected in this population. We aimed to fill this gap by investigating implicit learning and retention of statistical regularities in patients with BPD. Based on previous findings of implicit learning being less susceptible to illness (Reber and Allen, 2000; Reber, 2013), intact implicit learning and retention can be expected in BPD.

\section{Materials and methods}

\subsection{Participants}

Nineteen patients with borderline personality disorder (BPD group) were assessed on two consecutive days on the first week of their admission to an open ward for a 4-week-long inpatient program of cognitive behavior group therapy to the Department of Psychiatry and Psychotherapy, Semmelweis University, Budapest, Hungary. All 19 patients met DSM-IV criteria for BPD as assessed by the Structured Clinical Interview for DSM-IV Personality Disorders (SCID-II) (First et al., 1997a; Szádóczky et al., 2004). Axis I comorbidity was assessed with the Structured Clinical Interview (SCID-I) (First et al., 1997b; Szádóczky et al., 2004). All admitted patients with BPD who met the inclusion and exclusion criteria and were willing to participate were assessed.

Nineteen healthy control participants, recruited either from the community or from staff members of the Department, were matched pair-wise to patients based on age ( \pm 2 years), gender and years of education ( \pm 2 years). Exclusion criteria for the healthy control group included a current or lifetime diagnosis of any axis I or II disorder or a higher than 150 sum score of Symptom Checklist-90-Revised Hungarian version (Derogatis et al., 1977; Unoka et al., 2004). The SCL-90-R is a 90-item self-report questionnaire covering a wide range of psychopathological symptoms that are rated for severity with regard to the week prior to assessment. The Global Severity Index (sum score of all items) has been shown to have high predictive value regarding a statistically significant separation of clinical and normal samples (Unoka et al., 2004). Demographics, general cognitive functioning measured by the Digit Span (Isaacs and Vargha-Khadem, 1989; Racsmány et al., 2005) and Letter Fluency tasks (Spreen and Strauss, 1991; Tanczos et al., 2013), and clinical data, including state (STAI-S) and trait (STAIT) scores on State-Trait Anxiety Inventory (STAI) (Sipos and Sipos, 1983; Spielberger, 1970), are presented in Table 1. The STAI-S measures the transitional emotional status evoked by a stressful situation,
Table 1

Demographic data, general cognitive functions and clinical variables for the control and BPD groups. All participants were females.

\begin{tabular}{|c|c|c|c|c|c|}
\hline & \multicolumn{2}{|c|}{ Control } & \multicolumn{2}{|l|}{ BPD } & \multirow[b]{2}{*}{$p$-value } \\
\hline & Mean & $S D$ & Mean & $S D$ & \\
\hline Age (years) & 26.32 & 7.85 & 26.26 & 8.72 & 0.985 \\
\hline Education (years) & 15.42 & 1.98 & 14.05 & 2.23 & 0.053 \\
\hline Digit Span Task & 6.74 & 1.69 & 5.95 & 1.22 & 0.109 \\
\hline Letter Fluency Task & 20.34 & 7.33 & 17.17 & 4.06 & 0.115 \\
\hline STAI-T & 37.26 & 7.40 & 62.79 & 8.65 & $<0.001$ \\
\hline STAI-S & 32.84 & 6.87 & 56.16 & 13.17 & $<00.001$ \\
\hline Current Axis I diagnoses & & & $N$ & $\%$ & \\
\hline Major Depressive Disorder & & & 11 & 57.89 & \\
\hline Bipolar II disorder & & & 3 & 15.79 & \\
\hline \multicolumn{6}{|l|}{ (Current dysthymic episode) } \\
\hline Anxiety disorders & & & 10 & 52.63 & \\
\hline Substance abuse & & & 5 & 26.32 & \\
\hline Alcohol abuse & & & 4 & 21.05 & \\
\hline \multicolumn{6}{|l|}{ Anorexia Nervosa } \\
\hline Restrictive & & & 1 & 5.26 & \\
\hline Binge-purging & & & 3 & 15.79 & \\
\hline Bulimia nervosa & & & 3 & 15.79 & \\
\hline Somatization & & & 4 & 21.05 & \\
\hline \multicolumn{6}{|l|}{ Axis II diagnoses } \\
\hline Paranoid & & & 2 & 10.53 & \\
\hline Schizotypal & & & 2 & 10.53 & \\
\hline Depressive & & & 5 & 26.32 & \\
\hline Obsessive-compulsive & & & 2 & 10.53 & \\
\hline Avoidant & & & 5 & 26.32 & \\
\hline Dependent & & & 4 & 21.05 & \\
\hline Histrionic & & & 3 & 15.79 & \\
\hline Antisocial & & & 1 & 5.26 & \\
\hline
\end{tabular}

Note: STAI-S refers to state and STAI-T refers to trait scores on State-Trait Anxiety Inventory.

like participation in a study. The STAI-T score reflects relatively enduring individual differences in anxiety proneness. The validity and reliability of the STAI are well documented in the Hungarian population (Sipos and Sipos, 1983).

All participants gave written informed consent to participate in the study prior the assessment and gave their permission to use the data for research purposes. Participation in the study was voluntary, with no incentives offered. The study was approved by the local ethics committee of Semmelweis University. All subjects were Caucasian, had Hungarian as their native language, and had completed at least 8 years of education.

\subsection{Justification of the required sample size}

We calculated the required sample size based on previously published data where the exact same (5-epoch version) task was used to assess implicit statistical learning in Session 1, and retention was tested $24 \mathrm{~h}$ later in Session 2 (Nemeth and Janacsek, 2011). Since implicit statistical learning has not yet been tested in BPD before, we calculated required sample size assuming intact learning as it would be expected in a healthy young adult sample. G*Power 3.1.9.2 was used to calculate the effect size and the required sample size. Based on the mean and standard deviation of the learning score for Session 1 (i.e., difference between RT for high- vs. low-frequency triplets averaged across the five epochs, $M=8.81, S D=6.94, N=31$ participants), the estimated effect size was $d z=1.27$ (which corresponds to Cohen's $d=1.80$; for reference: Cohen's $d$ above 0.8 is considered a large effect size, (Cohen, 1977)). The required sample size to detect significant implicit statistical learning at $p=0.05$ and power $=0.80$ with an effect size of $d z=1.27$, is $N=6$ participants. Using stricter criteria of $p=0.01$ and power $=$ $0.95, N=15$ participants are required to detect the estimated effect. In the current study, we organized the data collection for 20 participants 
per group because in case some participants will drop out of the study or should be excluded because of outlier performance. Finally, only one participant had to be excluded from the study because of technical issues of data collection (received a different sequence in Session 2, thus, no retention could be tested), and the pairwise matched control participant was consequently also excluded. Although these calculations were based on a sample of healthy young adults, it is a reasonable assumption that even if the data of a patient group has a greater variability, such a strong learning effect (data of six healthy participants is sufficient to detect) could also be detected in the case of the BPD group with the sample size of the current study.

Regarding retention of statistical knowledge, Nemeth and Janacsek (2011) found no change in the learning score between the end of Session 1 and the Beginning of Session 2 (Epoch 5: $M=14.37, S D=9.73$, vs. Epoch 6: $M=12.97, S D=11.07$ ), the estimated effect size is negligible, not even reaching the small effect size category $(d z=$ 0.110). Based on this estimation of a nearly zero effect (and similar previous findings showing no change in the offline period, (Csabi et al., 2015; Csabi et al., 2014; Nemeth et al., 2010; Song et al., 2007)), we expected no change in statistical knowledge over the 24-h delay, and consequently, no required sample size was calculated here to detect a significant change. Instead, Bayes factor (BF) was computed on the collected data of the current study to determine whether there is enough evidence to accept the null-hypothesis of no offline change (for more details see the last paragraph of the section Statistical Analysis).

\subsection{The Alternating Serial Reaction Time (ASRT) task}

Implicit statistical learning was measured by the "Catch the dog" version (Nemeth et al., 2010) of the ASRT task (Howard and Howard, 1997). In this task, a stimulus (a dog's head) appears in one of four empty circles on the screen and participants have to press the corresponding button as fast and accurately as they can (Fig. 1A). The computer is equipped with a special keyboard which only contains four heightened keys ( $\mathrm{Z}, \mathrm{C}, \mathrm{B}$, and M on a QWERTY keyboard). These keys correspond to the target circles in a horizontal arrangement.

The appearance of stimuli follows a predetermined order, which stays unknown for the participants throughout the experiment. Stimuli are presented in blocks of 85 stimuli, from which the first five key presses are random for practice purposes. Then an 8-element alternating sequence (e.g., 2r4r3r1r, where numbers represents the four circles on the screen and ' $r$ ' represents randomly chosen locations out of the four possible locations) is repeated ten times in a block. Due to the structure of the sequences in the ASRT task, some triplets or runs of three consecutive events occur more frequently (high-frequency triplets) than others (low-frequency triplets). For example, in the above illustration, 2_4, 4_3, 3_1, and 1_2 (where "." indicates the middle element of the triplet) occur often because the third element (bold numbers) could be derived from the sequence or could also be a random element (Fig. 1B). In contrast, 1_3 or $4 \_1$ occur less frequently because in this case the third element could only be random. Note that the final event of high-frequency triplets is therefore more predictable from the initial event compared to the low-frequency triplets [also known as non-adjacent second-order dependency (Remillard, 2008)]. Therefore, each item can be coded as the last element of a high- or low-frequency triplet.

Overall, there are 64 possible versions of triplets $\left(4^{3}, 4\right.$ stimuli combined for three consecutive events) in the task, from which 16 are high-frequency triplets, each of them occurring on approximately $4 \%$ of the trials (62.5\% in total). Each of the remaining 48 triplets occurs on approximately $0.8 \%$ of the trials ( $37.5 \%$ in total). Thus, high-frequency triplets occur five times more often than low-frequency triplets. As people go further in practicing the ASRT task, they respond more quickly to the high- compared to the low-frequency triplets, revealing statistical learning (Howard and Howard, 1997; Song et al., 2007). In addition, general skill improvements also occur, which are observed in generally faster responses and changes in accuracy, independently of the triplet types (i.e., general skill improvements occur to a similar extent both in the case of high- and low-frequency triplets). These improvements reflect more efficient visuomotor coordination due to practice, thus, associating a given stimulus location with the corresponding response button, irrespective of whether that stimulus is the last element of a high- or a low-frequency triplet. While general skill improvements are evaluated by the changes (over the Learning Phase or over the offline period) in RT/accuracy averaged across high- and lowfrequency triplets, statistical learning is measured as RT/accuracy difference in responses to high- vs. low-frequency triplets during the Learning Phase or following the offline period (Nemeth et al., 2010; Song et al., 2007).

\subsection{Procedure}

The clinical assessment was made by a psychiatrist (Z.U.) and a clinical psychologist (G.V). The SCL-90-R were administered to the healthy controls before the inclusion in the study and to BPD patients at the admission to the ward.

The ASRT task was administered in two sessions. It consisted of 25 blocks in Session 1 (Learning Phase), which took approximately 25-35 min. Participants were informed that the main aim of the task was to investigate how extended practice affected performance on a simple reaction time task. Therefore, we emphasized them to perform the task as fast and as accurately as they could. They were not given any explicit information about the regularity of the sequence that was embedded in the task. Between blocks, they received feedback on the screen about their overall reaction time (RT) and accuracy, which was followed by a rest of 10-20 s before starting a new block. The ASRT task
A

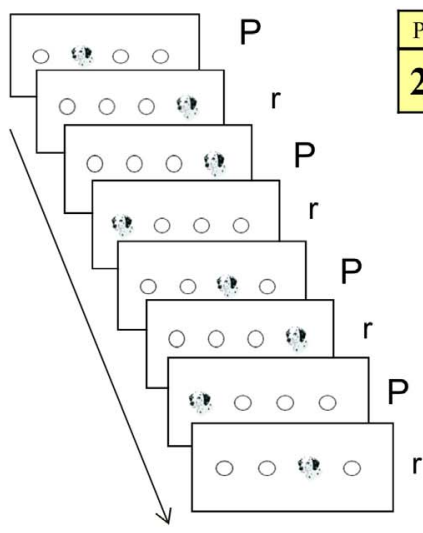

B

ASRT task: e.g., $2 \mathrm{r} 4 \mathrm{r} 3 \mathrm{r} 1 \mathrm{r}$

\begin{tabular}{|c|c|c|c|c|c|c|c|c|c|c|c|c|c|c|}
\hline $\mathrm{P}$ & $r$ & $P$ & $r$ & $P$ & $\mathrm{r}$ & $P$ & $\mathrm{r}$ & $P$ & $\mathrm{r}$ & $P$ & $\mathrm{r}$ & $P$ & $\mathrm{r}$ & $P$ \\
\hline 2 & \begin{tabular}{|l|l|}
1 & 2 \\
3 & 4 \\
\end{tabular} & 4 & \begin{tabular}{|l|l|}
1 & 2 \\
3 & 4 \\
\end{tabular} & 3 & \begin{tabular}{|l|l|}
1 & 2 \\
3 & 4 \\
\end{tabular} & & \begin{tabular}{|l|l|}
1 & 2 \\
3 & 4 \\
\end{tabular} & 2 & \begin{tabular}{|l|l|}
1 & 2 \\
3 & 4 \\
\end{tabular} & 4 & \begin{tabular}{|l|l|}
1 & 2 \\
3 & 4 \\
\end{tabular} & 3 & \begin{tabular}{|l|l|}
1 & 2 \\
3 & 4 \\
\end{tabular} & 1 \\
\hline & $\begin{array}{l}3- \\
\text { low fre } \\
\text { triplet }\end{array}$ & & & & $\begin{array}{l}\text { h frequ } \\
\text { let }(P-\end{array}$ & $\begin{array}{l}\text { uency } \\
-(-P)\end{array}$ & & & $\begin{array}{l}3- \\
\text { high fr } \\
\text { triple }\end{array}$ & & $\begin{array}{l}1 \\
\text { iency } \\
\text { P-r) }\end{array}$ & & & \\
\hline
\end{tabular}

\begin{tabular}{|c|c|c|}
\cline { 2 - 3 } \multicolumn{1}{c|}{} & $\begin{array}{c}\text { Structure: } \\
3-r-1\end{array}$ & $\begin{array}{c}\text { Structure: } \\
r-4-r\end{array}$ \\
\hline $\begin{array}{c}\text { High frequency } \\
\text { triplets } \\
(62.5 \% \text { of all trials) }\end{array}$ & $3-4-1(50 \%)$ & $3-4-1(12.5 \%)$ \\
\hline $\begin{array}{c}\text { Low frequency } \\
\text { triplets }\end{array}$ & $\begin{array}{c}\text { never occurring } \\
\text { (always high) }\end{array}$ & $\begin{array}{c}3-4-2(12.5 \%) \\
3-4-3(12.5 \%) \\
3-4-4(12.5 \%)\end{array}$ \\
\hline $\begin{array}{c}(37.5 \% \text { of all trials) } \\
\text { (alw }\end{array}$ & \\
\hline
\end{tabular}

Fig. 1. The Alternating Serial Reaction Time (ASRT) task. (A) In the ASRT task, a stimulus (a dog's head) appeared in one of four horizontally arranged empty circles on the screen. Participants were asked to press the button corresponding to the stimulus location (Z, C, B or M on a QWERTY keyboard). The presentation of stimuli followed an eight-element sequence, within which predetermined (P) and random ( $r$ ) elements alternated with each other. (B) The alternating sequence in the ASRT task makes some runs of three consecutive elements (triplets) more frequent than others. High frequency triplets are denoted with orange coloring and low frequency triplets are denoted with green coloring. (For interpretation of the references to color in this figure legend, the reader is referred to the web version of this article). 
A

Control

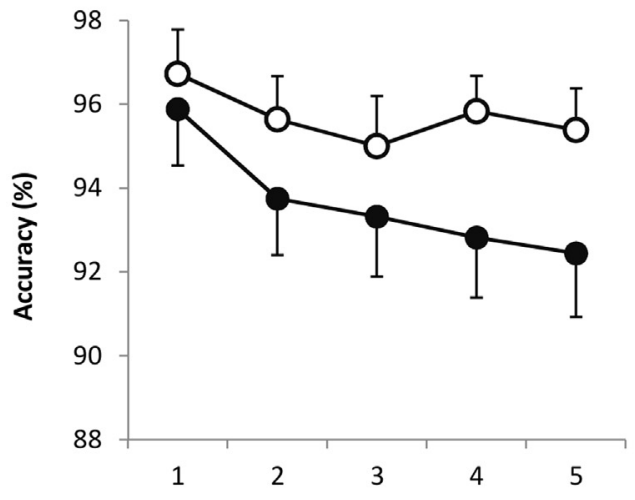

C

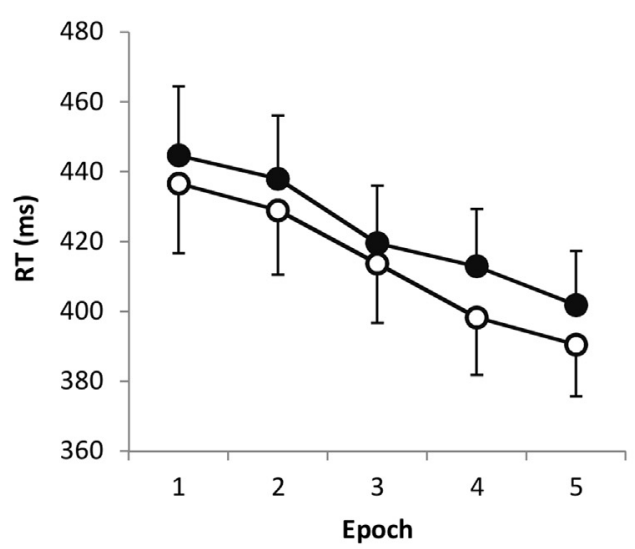

B

B BPD

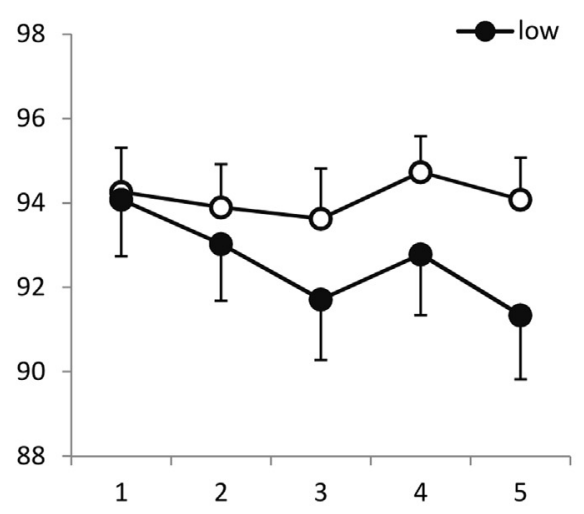

BPD

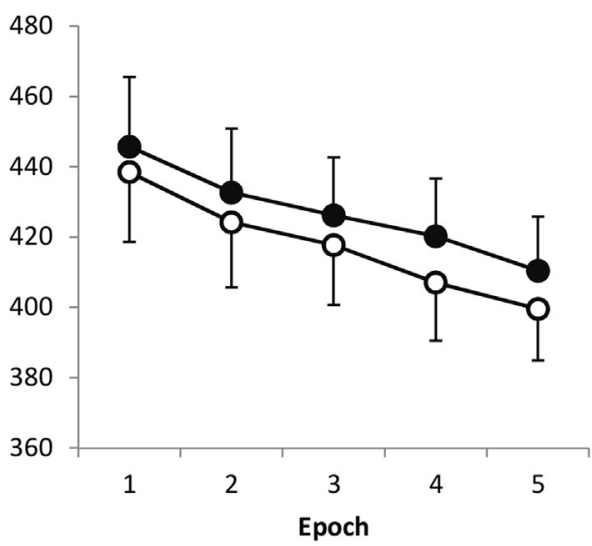

Fig. 2. Performance in the ASRT task during the Learning Phase (Session 1). Both the control and the BPD groups showed significant statistical learning in terms of accuracy (A-B) and reaction time (RT) (C-D), thus, they were more accurate on and responded faster to high-frequency triplets compared to the low-frequency ones. There were significant general skill improvements as well (i.e., RTs became faster during practice, to a similar extent in the case of high- and low-frequency triplets). No significant group differences were found. Error bars represent standard error of the mean (SEM). in Session 2 (Test Phase) consisted of 5 blocks only because here we aimed to test whether the acquired statistical knowledge in the Learning Phase was retained over a delay period. There was a 24-h delay between the two sessions.

The computer program selected a different ASRT sequence for each participant based on a permutation rule, such that each of the six unique permutations of the four possible stimuli occurred. Consequently, six different sequences were used across participants (Howard and Howard, 1997; Nemeth et al., 2010).

To explore how much explicit knowledge participants acquired about the sequential structure, we administered a short questionnaire after the ASRT task of Session 2 (Nemeth et al., 2010; Song et al., 2007). The questionnaire included increasingly specific questions such as "Have you noticed anything special regarding the task? Have you noticed some regularity in the sequence of stimuli?" The experimenter rated participants' answers on a 5-item scale, where 1 was "Nothing noticed" and 5 was "Total awareness". None of the participants in either the control or BPD group reported noticing the sequence in the task.

The digit span and letter fluency tests, and the STAI was administered after the explicit questionnaire in Session 2. All data were collected by two trained medical student experimenters.

\subsection{Statistical analysis}

Similarly to previous studies, two types of low-frequency triplets, repetitions (e.g., 222, 333) and trills (e.g., 212, 343), were eliminated because people often show preexisting response tendencies to them ( Howard et al., 2004; Soetens et al., 2004). By eliminating these triplets, we could ensure that any high- versus low-frequency differences were due to statistical learning and not to preexisting tendencies.

To facilitate data processing, the blocks of ASRT were organized into epochs of five blocks. The first epoch contained blocks $1-5$, the second blocks 6-10, etc. (Barnes et al., 2008; Bennett et al., 2007). We calculated mean accuracy and median RTs for correct responses only, separately for high- and low-frequency triplets and for each participant and each epoch. To evaluate statistical learning and its 24-h retention, we conducted mixed design analyses of variance (ANOVAs). Greenhouse-Geisser epsilon $(\varepsilon)$ correction was used when necessary. Original $d f$ values and corrected $p$ values (if applicable) are reported together with partial eta-squared $\left(\eta_{p}{ }^{2}\right)$ as the measure of effect size.

In addition to the classical statistical approach, we also performed Bayesian independent-samples $t$-tests and calculated the Bayes Factor (BF) for the relevant group comparisons (see the Results section below). The classical statistical approach relies on the p-value when rejecting the null-hypothesis $\left(H_{0}\right.$, i.e., no difference between groups or variables), for example at $p<0.05$, and accepting the alternative hypothesis $\left(H_{1}\right.$, i.e., significant differences between groups or variables). Importantly, if $p>0.05$, a non-significant result can mean one of two things: either that the null-hypothesis is true (no difference); or else that the data are insensitive in distinguishing the alternative- from the null-hypothesis and nothing follows from the data at all (Dienes, 2014, 2016). The BF is a statistical technique that helps conclude whether the collected data favors the null-hypothesis (i.e., evidence for no difference between groups or variables) or the alternative hypothesis (i.e., evidence for differences); thus, the BF could be considered as a weight of evidence provided by the data (Wagenmakers et al., 2011). Thus, one of the main benefits of calculating the $\mathrm{BF}$ is that for non-significant group comparisons we can use the $\mathrm{BF}$ to conclude that the two groups indeed do not differ, and the acquired evidence supports $H_{0}$ rather than $H_{1}$ (Dienes, 2011, 2014; Wagenmakers, 2007). BFs were calculated using the JASP version 0.6 (Rouder et al., 2009). Here we report $\mathrm{BF}_{10}$ values (read as "The Bayes Factor of $H_{1}$ against $H_{0}$ ", hence the 10 in the 
A Statistical regularities

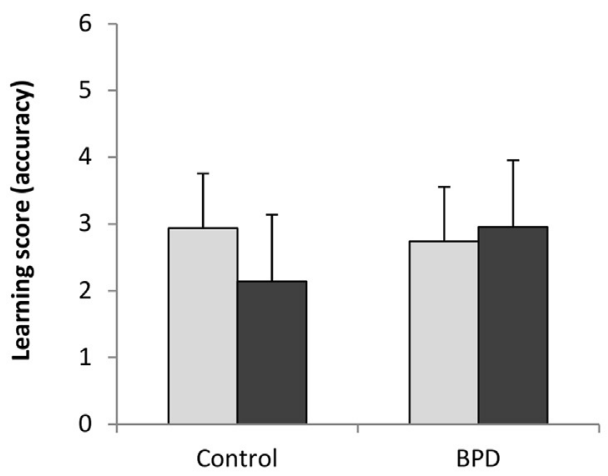

C Statistical regularities

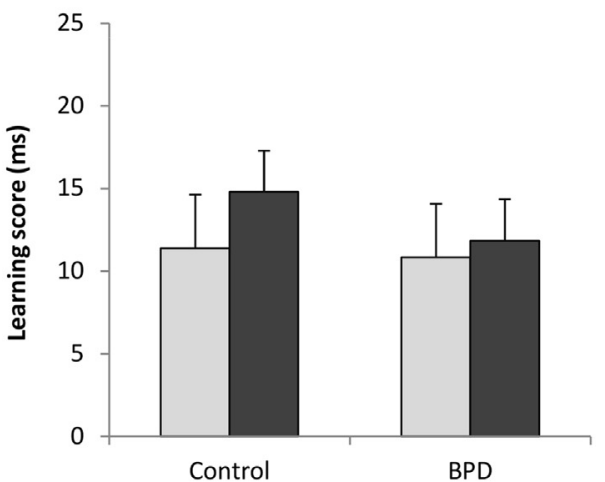

B

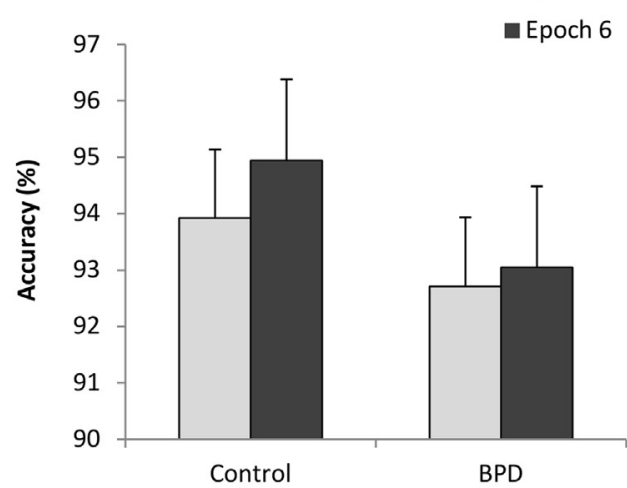

D

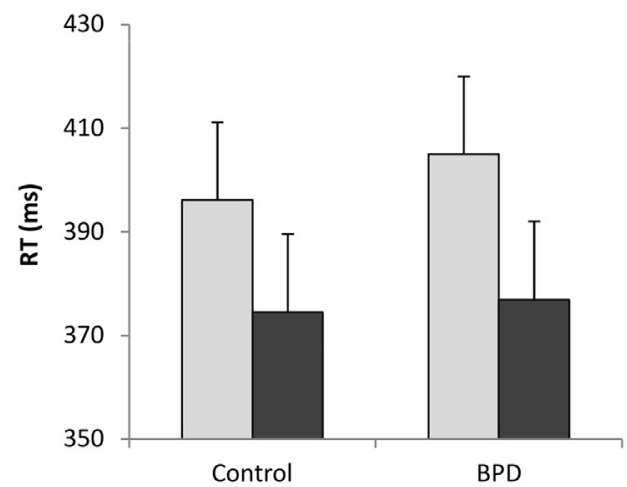

Fig. 3. Retention of the acquired knowledge over the 24-h delay period. Both the control and the BPD groups retained the acquired statistical knowledge (i.e., the difference score of high- vs. low-frequency triplets was similar in Epoch 5 and 6) in the case of accuracy (A) as well as in the case of reaction times (C). General skills were also retained; participants responded with similar accuracy in Epoch 6 as in Epoch 5 (B), plotted as averaging across high- and low-frequency triplets. Moreover, as it is typical in this task, participants were not only as fast in Epoch 6 as they were in Epoch 5 but became even faster (i.e., there was a significant general skill improvement in RTs in both groups over the 24-h delay period, plotted as averaging across high- and lowfrequency triplets; D). No significant group differences were found. Error bars represent SEM. subscript) where greater values support the alternative hypothesis (evidence for differences between groups or variables) over the nullhypothesis (evidence for no differences between groups or variables). According to Wagenmakers et al. (2011), $\mathrm{BF}_{10}$ values between 0.33 and 1 indicate anecdotal evidence for $H_{0}$, while values between 0.1 and 0.33 indicate substantial evidence for $H_{0}$. Conversely, while values between 1 and 3 indicate anecdotal evidence for $H_{1}$, values between 3 and 10 indicate substantial evidence for $H_{1}$. Values around one do not support either $H_{0}$ or $H_{1}$.

\section{Results}

\subsection{Do the BPD and control groups learn the statistical regularities} differently in terms of accuracy?

To compare statistical learning between the groups, accuracy was analyzed by a mixed design analysis of variance (ANOVA) with TRIPLET (2: high vs. low) and EPOCH (1-5) as within-subject factors and GROUP (control vs. BPD) as a between-subject factor (Fig. 2AB). Overall, participants in the control and BPD group responded with similar accuracy (main effect of GROUP: $F(1,36)=0.762, \eta_{\mathrm{p}}{ }^{2}=0.021$, $\left.p=0.389, \mathrm{BF}_{10}=0.425\right)$. Participants showed significant statistical learning, such that they responded more accurately to high-frequency triplets compared to the low-frequency ones (main effect of TRIPLET: $F$ $\left.(1,36)=32.721, \eta_{\mathrm{p}}{ }^{2}=0.476, p<0.001\right)$. The TRIPLET $\times$ GROUP interaction was not significant $\left(F(1,36)=0.753, \eta_{\mathrm{p}}{ }^{2}=0.020, p=\right.$ 0.391 ), suggesting that there was no difference between the control and BPD group in statistical learning. The Bayes Factor for the statistical learning score (accuracy for high- minus low-frequency triplets) for the entire Learning Phase compared over the two groups also tends to favor $H_{0}$ (no group difference) over $H_{1}\left(\mathrm{BF}_{10}=0.424\right)$.

Accuracy marginally decreased over epochs (main effect of EPOCH: $\left.F(4,144)=3.932, \eta_{\mathrm{p}}{ }^{2}=0.098, p=0.013\right)$, primarily due to increasing number of errors for low-frequency triplets as the task progressed, indicating that statistical learning increased during practice (TRIPLET $\times$ EPOCH interaction: $F(4,144)=3.732, \eta_{\mathrm{p}}{ }^{2}=0.094, p=$ 0.006). The EPOCH $\times$ GROUP and TRIPLET $\times$ EPOCH $\times$ GROUP interactions were not significant $\left(F(4,144)=0.531, \eta_{\mathrm{p}}{ }^{2}=0.015, p=\right.$ 0.646; $F(4,144)=0.338, \eta_{\mathrm{p}}{ }^{2}=0.009, p=0.852$, respectively), suggesting that the overall changes in accuracy and the dynamics of learning was similar in the two groups. To further support that accuracy decreased similarly in both groups, we compared mean accuracy in Epoch 1 minus accuracy in Epoch 5 over the two groups, and the BF also favored $H_{0}$ (no group difference) over $H_{1}\left(\mathrm{BF}_{10}=0.368\right)$.

\subsection{Do the BPD and control groups learn the statistical regularities differently in terms of reaction time?}

We conducted a similar ANOVA for RT data shown in Fig. 2CD with TRIPLET (2: high vs. low) and EPOCH (1-5) as within-subject factors and GROUP (control vs. BPD) as a between-subject factor. Overall, participants in the control and BPD group responded with similar RTs (main effect of GROUP: $F(1,36)=0.024, \eta_{\mathrm{p}}{ }^{2}=0.001, p=0.877, \mathrm{BF}_{10}$ $=0.318$ ). Participants showed significant statistical learning, such that they responded faster to high-frequency triplets compared to the lowfrequency ones (main effect of TRIPLET: $F(1,36)=48.198, \eta_{\mathrm{p}}{ }^{2}=$ $0.572, p<0.001)$. The TRIPLET $\times$ GROUP interaction was not significant $\left(F(1,36)=0.003, \eta_{\mathrm{p}}{ }^{2}<0.001, p=0.958\right)$, indicating that there was no difference between the control and BPD group in the amount of statistical learning. The Bayes Factor for the statistical learning score (RTs for low- minus high-frequency triplets) for the entire Learning Phase indicated substantial evidence for $H_{0}$ (no group difference) over $H_{1}\left(\mathrm{BF}_{10}=0.315\right)$.

In addition, RTs significantly decreased over epochs (main effect of EPOCH: $\left.F(4,144)=27.534, \eta_{\mathrm{p}}{ }^{2}=0.433, p<0.001\right)$, irrespectively of triplet type (TRIPLET $\times$ EPOCH interaction: $F(4,144)=1.807, \eta_{\mathrm{p}}{ }^{2}=$ $0.048, p=0.131$ ), showing general skill improvements. The EPOCH $\times$ GROUP and TRIPLET $\times$ EPOCH $\times$ GROUP interactions were not 
significant $\left(F(4,144)=0.837, \eta_{\mathrm{p}}^{2}=0.023, p=0.504 ; F(4,144)=\right.$ $0.147, \eta_{\mathrm{p}}^{2}=0.004, p=0.964$, respectively), suggesting that the general skill improvements as well as the overall dynamics of learning were similar in the two groups. We compared the decrease of RTs from Epoch 1 to Epoch 5 as a measure of general skill improvements, and the $\mathrm{BF}$ also tended to favor $H_{0}$ (no group difference) over $H_{1}\left(\mathrm{BF}_{10}=\right.$ 0.357).

\subsection{Do the BPD and control groups retain the acquired knowledge in terms of accuracy?}

To investigate the retention of statistical knowledge and general skills, participants' performance was retested in Session 2, which took place $24 \mathrm{~h}$ after Learning Phase (i.e., Session 1). Retention is defined as no change in performance between the end of Session 1 and the beginning of Session 2. Therefore, we tested retention by comparing accuracy from the last epoch of Session 1 (Epoch 5) and the epoch of Session 2 (Epoch 6; see Fig. 3AB). These variables were submitted to a mixed design ANOVA with TRIPLET (2: high- vs. low-frequency) and EPOCH (2: last epoch of Session 1 and epoch of Session 2) as withinsubject factors, and GROUP (control vs. BPD) as a between-subject factor.

Overall, participants in the control and BPD group responded with similar accuracy (main effect of GROUP: $F(1,36)=0.742, \eta_{\mathrm{p}}{ }^{2}=$ $0.020, p=0.395, \mathrm{BF}_{10}=0.422$ ). Accuracy did not change significantly during the 24-h delay period (main effect of EPOCH: $F(1,36)=1.436$, $\eta_{\mathrm{p}}{ }^{2}=0.038, p=0.239$ ), similarly in both groups (EPOCH $\times$ GROUP interaction: $\left.F(1,36)=0.368, \eta_{\mathrm{p}}{ }^{2}=0.010, p=0.548\right)$. The BF also tended to favor $H_{0}$ (no group difference) over $H_{1}$ as overall accuracy in Epoch 5 minus overall accuracy in Epoch 6 was compared over the two groups $\left(\mathrm{BF}_{10}=0.364\right)$.

The main effect of TRIPLET was significant $\left(F(1,36)=22.019, \eta_{\mathrm{p}}{ }^{2}\right.$ $=0.380, p<0.001$ ), indicating statistical knowledge with more accurate responses on high- than on low-frequency triplets, similarly in both groups (TRIPLET $\times$ GROUP interaction: $F(1,36)=0.072, \eta_{\mathrm{p}}{ }^{2}=$ $0.002, p=0.789$ ). This knowledge did not change significantly during the delay period (TRIPLET $\times$ EPOCH interaction: $F(1,36)=0.237, \eta_{\mathrm{p}}{ }^{2}$ $=0.007, p=0.629$ ), both the control and BPD groups retained the acquired statistical knowledge (indicated by the non-significant TRIPLET $\times$ EPOCH $\times$ GROUP interaction: $F(1,36)=0.709, \eta_{\mathrm{p}}{ }^{2}=$ $0.019, p=0.405$ ). The $\mathrm{BF}$ also tended to favor no group differences in the retention of the acquired statistical knowledge (statistical learning score in Epoch 5 minus statistical learning score in Epoch 6 compared over the two groups; $\mathrm{BF}_{10}=0.416$ ).

3.4. Do the BPD and control groups retain the acquired knowledge in terms of reaction time?

Similarly to the accuracy analysis, RT data shown in Fig. 3CD were submitted to a mixed design ANOVA with TRIPLET (2: high- vs. lowfrequency) and EPOCH (2: last epoch of Session 1 and epoch of Session 2) as within-subject factors, and GROUP (control vs. BPD) as a betweensubject factor.

Overall, participants in the control and BPD groups responded with similar RTs (main effect of GROUP: $F(1,36)=0.071, \eta_{\mathrm{p}}{ }^{2}=0.002, p$ $\left.=0.792, \mathrm{BF}_{10}=0.324\right)$. RTs significantly decreased during the 24-h delay period (main effect of EPOCH: $F(1,36)=99.336, \eta_{\mathrm{p}}{ }^{2}=0.734$, $p<0.001$ ), such that participants were faster in Session 2 compared to the end of Session 1, indicating offline general skill improvements, which is typical in these types of tasks. The degree of this improvement over the offline period was similar in the two groups $(\mathrm{EPOCH} \times$ GROUP interaction: $\left.F(1,36)=1.648, \eta_{\mathrm{p}}^{2}=0.044, p=0.207\right)$. The BF also tended to favor $H_{0}$ (no group difference) over $H_{1}\left(\mathrm{BF}_{10}=0.600\right.$ ), although the slightly weaker BF could be attributed to even larger speed-up in the BPD group compared to the controls (28 vs. $21 \mathrm{~ms}$, respectively).
The main effect of TRIPLET was significant $\left(F(1,36)=50.140, \eta_{\mathrm{p}}{ }^{2}\right.$ $=0.582, p<0.001$ ), indicating statistical knowledge with faster responses on high- than on low-frequency triplets, similarly in both groups (TRIPLET $\times$ GROUP interaction: $F(1,36)=0.257, \eta_{\mathrm{p}}{ }^{2}=0.007$, $p=0.615$ ). This knowledge did not change significantly during the delay period (TRIPLET $\times$ EPOCH interaction: $F(1,36)=0.992, \eta_{\mathrm{p}}{ }^{2}=$ 0.027, $p=0.326$ ), both the control and BPD groups showed retention of the statistical knowledge acquired in Session 1 (suggested by the nonsignificant TRIPLET $\times$ EPOCH $\times$ GROUP interaction: $F(1,36)=$ $0.294, \eta_{\mathrm{p}}{ }^{2}=0.008, p=0.591$ ). Similar level of retention of the acquired statistical knowledge was further supported by the BF (statistical learning score in Epoch 5 minus statistical learning score in Epoch 6 compared over the two groups; $\mathrm{BF}_{10}=0.354$ ).

\subsection{Is implicit learning performance related to the level of anxiety and performance on other cognitive tasks in the current sample?}

We run correlation analyses to explore the potential relationship between digit span and verbal fluency task performance, on one hand, and implicit learning and retention, on the other hand. In addition, we also explored potential associations between STAI and implicit learning scores. In the case of implicit learning, both accuracy and RT learning measures (difference scores between high- and low-frequency triplets for Epoch 5 and 6, plotted in Fig. 3) were entered in the analysis. We found no significant correlation between digit span/fluency performance and implicit learning scores either in the case of accuracy or RT, and either for the BPD or the control group (accuracy, BPD group: all ps $>0.357$; accuracy, controls: all $p s>0.313$; RT, BPD group: all ps $>0.212$; RT, controls: all ps $>0.516$ ). Similarly, we found no significant correlation between STAI scores and learning measures either (accuracy, BPD group: all $p s>0.330$; accuracy, controls: all ps $>0.289$; RT, BPD group: all $p s>0.141$; RT, controls: all ps $>0.137)$.

\section{Discussion}

Our main goal was to investigate how borderline personality disorder impacts implicit statistical learning. Based on the classical statistical approach, we did not find statistically significant difference between the learning performance in BPD patients and in controls either in terms of accuracy or reaction time. Importantly, Bayes factors (BFs) were also calculated to test whether our data favors the null-hypothesis (evidence for no group difference) or the alternative hypothesis (evidence for group difference). Based on the BFs, our data provides evidence for a similar learning performance in BPD patients and in controls (i.e., no group differences) both in terms of accuracy and reaction time. Independently of statistical learning, general skill improvements were also intact in BPD (evidence for no group differences based on the BF values). Moreover, the acquired statistical knowledge as well as general skills were retained over a 24-h delay period both in BPD and control groups (as confirmed by the BF values). These results altogether indicate effective implicit statistical learning in BPD. Importantly, our findings are supported by Bayes factors, corroborating the classical statistical approach.

Since this is, to the best of our knowledge, the first study investigating implicit learning in $\mathrm{BPD}$, we cannot compare our findings to similar studies. However, other aspects of learning and memory were investigated in $\mathrm{BPD}$, and previous research found disturbances in shortterm memory as well as in declarative and emotional learning and memory (Ebner-Priemer et al., 2009; Richman and Unoka, 2015). A dissociation between implicit learning/retention and other forms of learning/memory has been previously shown in other clinical populations, showing intact implicit learning/retention and impairments in short-term, working and declarative memory in the same group of patients (e.g., patients with sleep disorders or alcohol-dependency, (Csabi et al., 2015; Virag et al., 2015)). Those findings suggest that implicit 
learning/memory might be less susceptible to illness compared to other forms of learning/memory. Nevertheless, in the case of BPD, future studies should explore such a potential dissociation by administering a wide range of tests measuring both declarative and non-declarative forms of learning/memory, in the same group of participants.

The main goal of this study was to assess implicit learning and retention in BPD, and the required sample size was planned accordingly. Nevertheless, we run exploratory correlational analyses to examine a potential relationship between performance on other cognitive tasks (digit span and verbal fluency task) and level of anxiety (STAI scores), on one hand, and implicit learning and retention, on the other hand. We found no significant correlation among these measures either for the BPD or the control group. It is possible that the current sample size is not sufficient to find associations among these measures. It is still debated if relationship exists between short-term memory (STM), executive functions and implicit sequence/statistical learning (Janacsek and Nemeth, 2013, 2015; Martini et al., 2014), and if does then what its direction is. Several studies claim a competitive relationship between frontal lobe-related control/executive functions and implicit learning (Filoteo et al., 2010; Nemeth et al., 2013; Poldrack et al., 2001; Poldrack and Packard, 2003), and consequently, negative correlation was found between executive functions and implicit statistical learning (Nemeth et al., 2013; Virag et al., 2015). Future studies should directly focus on these potential associations in BPD with a larger sample size.

In the current study the BPD group differed significantly from the healthy controls in the level of anxiety. The question could be raised: does the different level of anxiety affect the observed results? Importantly, we found intact implicit statistical learning and retention in BPD patients despite their higher anxiety level. Thus, we can claim that at least in this case - higher level of anxiety did not lead to weaker learning or retention performance. Remarkably, a growing body of evidence suggests that higher level of stress and anxiety in fact alters the balance between frontal-lobe dependent explicit/declarative functions, and implicit/non-declarative functions, and under stress/higher anxiety people tend to rely more on implicit/non-declarative functions (Schwabe and Wolf, 2012, 2013). About the potential mediating mechanism: it has been found that anxiety/stress limits the resources of executive functions and STM (Eysenck et al., 2007; Klein and Boals, 2001; Oei et al., 2006), which also creates a limitation in explicit/declarative learning and memory as these functions rely on executive/ control functions and STM more heavily compared to implicit/nondeclarative functions (Becker and Lim, 2003; Simons and Spiers, 2003; Takashima et al., 2006). Notably, these findings are also in line with the negative, competitive relationship discussed in the previous paragraph.

A limitation of the study is that we used only one task to assess implicit learning and retention. There are several tasks that can be used for these purposes; for example, the Weather Prediction task, the Artificial Grammar Learning task and the classical Serial Reaction Time (SRT) task with deterministic sequences (Reber, 2013). Nevertheless, we decided to use the ASRT task because of the following reasons: compared to the other types of tasks, 1) it is well documented that participants do not become aware of the underlying sequence/statistical structure embedded in the ASRT task even after extended practice (e.g., ten days; (Howard et al., 2004)) and when examined with more sensitive recognition tests (Song et al., 2007), thus it indeed measures implicit learning; 2) ASRT enables us to continuously measure learning performance from the very beginning of the task through a longer learning session (250 sequence presentations in the current study); and 3 ) it also enables to separately measure general skill improvements and learning of statistical regularities (Janacsek and Nemeth, 2012). There are also various tasks to assess statistical learning (irrespective of whether it is learned implicitly or with explicit awareness). One main difference in these tasks is whether the elements of the structure-to-belearned is temporally distributed as in the case of ASRT (i.e., participants see only one stimulus at a time, and have to find the associations between subsequent stimuli by binding them across time) vs. in a Visual
Statistical Learning task (Fiser and Aslin, 2002) all relevant stimuli are presented at the same time that might help participants to find the associations faster. Future studies are needed to replicate our findings with other tasks, while also keeping in mind these differences among them, and carefully select the task depending on the exact research question (e.g., learning temporally vs. spatially distributed associations). It might also be possible that BPD patients might have difficulty learning other types of associations. Nevertheless, in the current study BPD patients showed intact learning of temporally distributed statistical regularities and intact retention of the acquired knowledge over the 24h delay.

Based on our results, BPD patients seem to be able to extract and represent regularities among neutral stimuli and to build a mental model of the environment by implicit statistical learning. As they have difficulties primarily in the social domain, it would be important to test whether and how BPD patients can extract and represent regularities among elements/events if these elements have social meaning. It is possible that in this case weaker learning performance would be found compared to the performance of healthy controls. On the other hand, it is possible that suffering from BPD might not be explained by the learning process itself (whether the stimuli have a social relevance of not) but the failure to overwrite ("rewire") the already existing knowledge. When the environmental regularities are changed, the inability to successfully detect these changes and update their models can lead to inflexible and rigid behavior. Further studies need to investigate this possibility.

\section{Declaration of interest}

No conflict of interest. All authors report no biomedical financial interests or potential conflicts of interest.

\section{Acknowledgements}

This research was supported by the Hungarian Brain Research Program (KTIA NAP 13-2-2015-0002), and Janos Bolyai Research Fellowship of the Hungarian Academy of Sciences (to K.J.).

\section{References}

APA, 2013. Diagnostic and Statistical Manual of Mental Disorders (DSM-5 ${ }^{\circledR}$ ): American Psychiatric Pub.

Barnes, K.A., Howard Jr., J.H., Howard, D.V., Gilotty, L., Kenworthy, L., Gaillard, W.D., Vaidya, C.J., 2008. Intact implicit learning of spatial context and temporal sequences in childhood autism spectrum disorder. Neuropsychology 22 (5), 563-570.

Beblo, T., Saavedra, A.S., Mensebach, C., Lange, W., Markowitsch, H.-J., Rau, H., Driessen, M., 2006. Deficits in visual functions and neuropsychological inconsistency in Borderline Personality Disorder. Psychiatry Res. 145 (2), 127-135.

Becker, S., Lim, J., 2003. A computational model of prefrontal control in free recall: strategic memory use in the California Verbal Learning Task. J. Cogn. Neurosci. 15 (6), 821-832.

Bennett, I.J., Howard Jr., J.H., Howard, D.V., 2007. Age-related differences in implicit learning of subtle third-order sequential structure. J. Gerontol.: Psychol. Sci. 62B (2), 98-103.

Black, D.W., Forbush, K.T., Langer, A., Shaw, M., Graeber, M.A., Moser, D.J., Meyer, V.J., 2009. The neuropsychology of borderline personality disorder: a preliminary study on the predictive variance of neuropsychological tests vs. personality trait dimensions. Personal. Ment. Health 3 (2), 128-141.

Cohen, J., 1977. Statistical Power Analysis for the Behavioral Sciences. Academic Press, New York.

Coid, J., Yang, M., Tyrer, P., Roberts, A., Ullrich, S., 2006. Prevalence and correlates of personality disorder in Great Britain. Br. J. Psychiatry 188 (5), 423-431.

Csabi, E., Varszegi-Schulz, M., Janacsek, K., Malecek, N., Nemeth, D., 2014. The consolidation of implicit sequence memory in obstructive sleep apnea. PloS One 9 (10), e109010.

Csabi, E., Benedek, P., Janacsek, K., Zavecz, Z., Katona, G., Nemeth, D., 2015. Declarative and non-declarative memory consolidation in children with sleep disorder. Front. Human. Neurosci. 9, 709. http://dx.doi.org/10.3389/fnhum.2015.00709.

Daw, N.D., Niv, Y., Dayan, P., 2005. Uncertainty-based competition between prefrontal and dorsolateral striatal systems for behavioral control. Nat. Neurosci. 8 (12), 1704-1711.

Derogatis, L.R., Lipman, R., Covi, L., 1977. SCL-90. Administration, Scoring and Procedures Manual-i for the R (Revised) Version and Other Instruments of the 
Psychopathology Rating Scales Series. Johns Hopkins University School of Medicine, Chicago.

Dienes, Z., 2011. Bayesian versus orthodox statistics: which side are you on? Perspect. Psychol. Sci. 6 (3), 274-290. http://dx.doi.org/10.1177/1745691611406920.

Dienes, Z., 2014. Using Bayes to get the most out of non-significant results. Front. Psychol. 5. http://dx.doi.org/10.3389/fpsyg.2014.00781.

Dienes, Z., 2016. How Bayes factors change scientific practice. J. Math. Psychol. 72, 78-89.

Doyon, J., Bellec, P., Amsel, R., Penhune, V., Monchi, O., Carrier, J., Benali, H., 2009. Contributions of the basal ganglia and functionally related brain structures to motor learning. Behav. Brain Res. 199 (1), 61-75.

Ebner-Priemer, U.W., Mauchnik, J., Kleindienst, N., Schmahl, C., Peper, M., Rosenthal, M.Z., Bohus, M., 2009. Emotional learning during dissociative states in borderline personality disorder. J. Psychiatry Neurosci.: JPN 34 (3), 214.

Eysenck, M.W., Derakshan, N., Santos, R., Calvo, M.G., 2007. Anxiety and cognitive performance: attentional control theory. Emotion 7 (2), 336.

Filoteo, J.V., Lauritzen, S., Maddox, W.T., 2010. Removing the frontal lobes the effects of engaging executive functions on perceptual category learning. Psychol. Sci. 21 (3), 415-423.

First, M.B., Gibbon, M., Spitzer, R.L., Benjamin, L.S., 1997a. User's guide for the structured clinical interview for DSM-IV axis II personality disorders: SCID-II: American Psychiatric Pub.

First, M.B., Spitzer, R.L., Gibbon, M., Williams, J.B., 1997b. User's guide for the structured clinical interview for DSM-IV axis I disorders SCID-I: clinician version: American Psychiatric Pub.

Fiser, J., Aslin, R.N., 2002. Statistical learning of higher-order temporal structure from visual shape sequences. J. Exp. Psychol. Learn., Mem., Cogn. 28 (3), 458-467.

Grant, B.F., Chou, S.P., Goldstein, R.B., Huang, B., Stinson, F.S., Saha, T.D., Pickering, R.P., 2008. Prevalence, correlates, disability, and comorbidity of DSM-IV borderline personality disorder: results from the Wave 2 National Epidemiologic Survey on Alcohol and Related Conditions. J. Clin. Psychiatry 69 (4), 533.

Gunderson, J.G., Stout, R.L., McGlashan, T.H., Shea, M.T., Morey, L.C., Grilo, C.M., Sanislow, C., 2011. Ten-year course of borderline personality disorder: psychopathology and function from the Collaborative Longitudinal Personality Disorders study. Arch. General. Psychiatry 68 (8), 827-837.

Haaland, V., Landrø, N.I., 2007. Decision making as measured with the Iowa Gambling Task in patients with borderline personality disorder. J. Int. Neuropsychol. Soc. 13 (04), 699-703.

Haaland, V., Esperaas, L., Landrø, N., 2009. Selective deficit in executive functioning among patients with borderline personality disorder. Psychol. Med. 39 (10), 1733-1743.

Hikosaka, O., Nakahara, H., Rand, M.K., Sakai, K., Lu, X., Nakamura, K., Doya, K., 1999. Parallel neural networks for learning sequential procedures. Trends Neurosci. 22 (10), 464-471.

Howard, D.V., Howard Jr., J.H., Japikse, K.C., DiYani, C., Thompson, A., Somberg, R., 2004. Implicit sequence learning: effects of level of structure, adult age, and extended practice. Psychol. Aging.

Howard Jr., J.H., Howard, D.V., 1997. Age differences in implicit learning of higher-order dependencies in serial patterns. Psychol. Aging 12 (4), 634-656. http://dx.doi.org/ 10.1037/0882-7974.12.4.634.

Isaacs, E.B., Vargha-Khadem, F., 1989. Differential course of development of spatial and verbal memory span: a normative study. Br. J. Dev. Psychol. 7 (4), 377-380.

Janacsek, K., Nemeth, D., 2012. Predicting the future: from implicit learning to consolidation. Int. J. Psychophysiol. 83 (2), 213-221.

Janacsek, K., Nemeth, D., 2013. Implicit sequence learning and working memory: correlated or complicated? Cortex 49 (8), 2001-2006.

Janacsek, K., Nemeth, D., 2015. The puzzle is complicated: when should working memory be related to implicit sequence learning, and when should it not?(Response to Martini et al.). Cortex 64, 411-412.

Janacsek, K., Fiser, J., Nemeth, D., 2012. The best time to acquire new skills: age-related differences in implicit sequence learning across the human lifespan. Dev. Sci. 15 (4), 496-505. http://dx.doi.org/10.1111/j.1467-7687.2012.01150.

Kaufman, S.B., DeYoung, C.G., Gray, J.R., Jiménez, L., Brown, J., Mackintosh, N., 2010. Implicit learning as an ability. Cognition 116 (3), 321-340.

Klein, K., Boals, A., 2001. The relationship of life event stress and working memory capacity. Appl. Cogn. Psychol. 15 (5), 565-579.

LeGris, J., Links, P.S., van Reekum, R., Tannock, R., Toplak, M., 2012. Executive function and suicidal risk in women with borderline personality disorder. Psychiatry Res. 196 (1), 101-108.

Lieberman, M.D., 2000. Intuition: a social cognitive neuroscience approach. Psychol. Bull. 126 (1), 109-137.

Martini, M., Sachse, P., Furtner, M.R., Gaschler, R., 2014. Why should working memory be related to incidentally learned sequence structures? Cortex.

Minzenberg, M.J., Poole, J.H., Vinogradov, S., 2008. A neurocognitive model of borderline personality disorder: effects of childhood sexual abuse and relationship to adult social attachment disturbance. Dev. Psychopathol. 20 (1), 341.

Nemeth, D., Janacsek, K., 2011. The dynamics of implicit skill consolidation in young and elderly adults. J. Gerontol. Psychol. Sci. 66 (1), 15-22.

Nemeth, D., Janacsek, K., Londe, Z., Ullman, M.T., Howard, D.V., Howard Jr., J.H., 2010. Sleep has no critical role in implicit motor sequence learning in young and old adults. Exp. Brain Res. 201 (2), 351-358. http://dx.doi.org/10.1007/s00221-009-2024-x.

Nemeth, D., Janacsek, K., Csifcsak, G., Szvoboda, G., Howard Jr., J.H., Howard, D.V., 2011. Interference between sentence processing and probabilistic implicit sequence learning. PloS One 8 (6(3)), e17577.

Nemeth, D., Janacsek, K., Polner, B., Kovacs, Z.A., 2013. Boosting human learning by hypnosis. Cereb. Cortex 23 (4), 801-805. http://dx.doi.org/10.1093/cercor/bhs068.
Norman, E., Price, M.C., 2012. Social intuition as a form of implicit learning: sequences of body movements are learned less explicitly than letter sequences. Adv. Cogn. Psychol. $8(2), 121-131$.

Oei, N.Y., Everaerd, W.T., Elzinga, B.M., van Well, S., Bermond, B., 2006. Psychosocial stress impairs working memory at high loads: an association with cortisol levels and memory retrieval. Stress 9 (3), 133-141.

Poldrack, R.A., Packard, M.G., 2003. Competition among multiple memory systems: converging evidence from animal and human brain studies. Neuropsychologia 41 (3), 245-251.

Poldrack, R.A., Clark, J., Pare-Blagoev, E.J., Shohamy, D., Creso Moyano, J., Myers, C., Gluck, M.A., 2001. Interactive memory systems in the human brain. Nature 414 (6863), 546-550.

Poldrack, R.A., Sabb, F.W., Foerde, K., Tom, S.M., Asarnow, R.F., Bookheimer, S.Y., Knowlton, B.J., 2005. The neural correlates of motor skill automaticity. Journal of Neuroscience 25. pp. 5356-5364.

Racsmány, M., Lukács, Á., Németh, D., Pléh, C., 2005. A verbális munkamemória magyar nyelvû́ vizsgálóeljárásai (Hungarian diagnostic tools of verbal working memory functions). Magy. Pszichol. Szle. (Hung. Rev. Psychol.) 60 (4), 479-506.

Reber, A.S., 1993. Implicit Learning and Tacit Knowledge: An Essay on the Cognitive Unconscious 19 Oxford University Press, New York.

Reber, A.S., Allen, R., 2000. Individual differences in implicit learning: Implications for the evolution of consciousness. 227.

Reber, P.J., 2013. The neural basis of implicit learning and memory: a review of neuropsychological and neuroimaging research. Neuropsychologia 51 (10), 2026-2042.

Rebuschat, P., 2013. Measuring implicit and explicit knowledge in second language research. Lang. Learn. 63 (3), 595-626.

Reed, L.I., Fitzmaurice, G., Zanarini, M., 2012a. The course of positive affective and cognitive states in borderline personality disorder: a 10-year follow-up study. Personal. Ment. Health 6 (4), 281-291.

Reed, L.I., Fitzmaurice, G., Zanarini, M.C., 2012b. The course of dysphoric affective and cognitive states in borderline personality disorder: a 10-year follow-up study. Psychiatry Res. 196 (1), 96-100.

Remillard, G., 2008. Implicit learning of second-, third-, and fourth-order adjacent and nonadjacent sequential dependencies. Q. J. Exp. Psychol. 61 (3), 400-424. http://dx. doi.org/10.1080/17470210701210999.

Rentrop, M., Backenstrass, M., Jaentsch, B., Kaiser, S., Roth, A., Unger, J., Renneberg, B., 2007. Response inhibition in borderline personality disorder: performance in a Go/ Nogo task. Psychopathology 41 (1), 50-57.

Richman, M.J., Unoka, Z., 2015. Mental state decoding impairment in major depression and borderline personality disorder: meta-analysis. Br. J. Psychiatry 207 (6), 483-489.

Rohrmeier, M., Rebuschat, P., 2012. Implicit learning and acquisition of music. Top. Cogn. Sci. 4 (4), 525-553.

Romano Bergstrom, J.C., Howard Jr., J.H., Howard, D.V., 2012. Enhanced implicit sequence learning in college-age video game players and musicians. Appl. Cogn. Psychol. 26 (1), 91-96.

Rouder, J.N., Speckman, P.L., Sun, D., Morey, R.D., Iverson, G., 2009. Bayesian t tests for accepting and rejecting the null hypothesis. Psychon. Bull. Rev. 16 (2), 225-237. http://dx.doi.org/10.3758/pbr.16.2.225.

Ruocco, A.C., 2005. The neuropsychology of borderline personality disorder: a metaanalysis and review. Psychiatry Res. 137 (3), 191-202.

Sadikaj, G., Russell, J.J., Moskowitz, D., Paris, J., 2010. Affect dysregulation in individuals with borderline personality disorder: persistence and interpersonal triggers. J. Personal. Assess. 92 (6), 490-500.

Schwabe, L., Wolf, O.T., 2012. Stress modulates the engagement of multiple memory systems in classification learning. J. Neurosci. 32 (32), 11042-11049. http://dx.doi. org/10.1523/JNEUROSCI.1484-12.2012.

Schwabe, L., Wolf, O.T., 2013. Stress and multiple memory systems: from 'thinking'to 'doing'. Trends Cogn. Sci. 17 (2), 60-68.

Seres, I., Unoka, Z., Keri, S., 2009. The broken trust and cooperation in borderline personality disorder. Neuroreport 20 (4), 388-392.

Simons, J.S., Spiers, H.J., 2003. Prefrontal and medial temporal lobe interactions in longterm memory. Nat. Rev. Neurosci. 4 (8), 637-648.

Sipos, K., Sipos, M., 1983. The development and validation of the Hungarian Form of the State-Trait Anxiety Inventory. Series in Clinical \& Community Psychology: Stress \& Anxiety.

Soetens, E., Melis, A., Notebaert, W., 2004. Sequence learning and sequential effects. Psychol. Res. 69 (1), 124-137.

Song, S., Howard Jr., J.H., Howard, D.V., 2007. Sleep does not benefit probabilistic motor sequence learning. J. Neurosci. 27 (46), 12475-12483. http://dx.doi.org/10.1523/ JNEUROSCI.2062-07.2007.

Spielberger, C.D., 1970. STAI manual for the state-trait anxiety inventory. Self-Eval. Quest. 1-24.

Spreen, O., Strauss, E., 1991. Language tests. Compend. Neuropsychol. Tests 268-275.

Stepp, S.D., Pilkonis, P.A., Yaggi, K.E., Morse, J.Q., Feske, U., 2009. Interpersonal and emotional experiences of social interactions in borderline personality disorder. J. Nerv. Ment. Dis. 197 (7), 484

Szádóczky, E., Unoka, Z., Rózsa, S., 2004. User's guide for the structured clinical interview for DSM-IV axis II personality disorders (SCID-II), Hungarian Version. OS Hungary Kft.

Takashima, A., Jensen, O., Oostenveld, R., Maris, E., Van De Coevering, M., Fernández, G., 2006. Successful declaritive memory formation is associated with ongoing activity during encoding in a distributed neocortical network related to working memory: a magnetoencephalography study. Neuroscience 139, 291-297.

Tanczos, T., Janacsek, K., Nemeth, D., 2013. [Verbal fluency tasks I. investigation of the Hungarian version of the letter fluency task between 5 and 89 years of age]. 
Psychiatr. Hung.: A Magy. Pszichiatr. Tars. Tud. F. 29 (2), 158-180.

Trull, T.J., Jahng, S., Tomko, R.L., Wood, P.K., Sher, K.J., 2010. Revised NESARC personality disorder diagnoses: gender, prevalence, and comorbidity with substance dependence disorders. J. Personal. Disord. 24 (4), 412-426.

Unoka, Z., Richman, M.J., 2016. Neuropsychological deficits in BPD patients and the moderator effects of co-occurring mental disorders: a meta-analysis. Clin. Psychol. Rev. 44, 1-12.

Unoka, Z., Rózsa, S., Kő, N., Kállai, J., Fábián, Á., Simon, L., 2004. Validity and reliability of the SCL-90 in a Hungarian population sample. Psychiatr. Hung. 19, 235-243.

Unoka, Z., Fogd, D., Füzy, M., Csukly, G., 2011. Misreading the facial signs: specific impairments and error patterns in recognition of facial emotions with negative valence in borderline personality disorder. Psychiatry Res. 189 (3), 419-425.

Virag, M., Janacsek, K., Horvath, A., Bujdoso, Z., Fabo, D., Nemeth, D., 2015. Competition between frontal lobe functions and implicit sequence learning: evidence from the long-term effects of alcohol. Exp. Brain Res. 233 (7), 2081-2089.

Wagenmakers, E.J., 2007. A practical solution to the pervasive problems of p values. Psychon. Bull. Rev. 14 (5), 779-804. http://dx.doi.org/10.3758/BF03194105.

Wagenmakers, E.J., Wetzels, R., Borsboom, D., van der Maas, H.L., 2011. Why psychologists must change the way they analyze their data: the case of psi: comment on Bem (2011). J. Personal. Social. Psychol. 100 (3), 426-432. http://dx.doi.org/10.1037/ a0022790. 